\title{
More on nano semi pre-regular and nano semi pre normal-spaces
}

\author{
P. Sathishmohan ${ }^{1^{*}}$, V. Rajendran ${ }^{2}$ and C. Vignesh Kumar ${ }^{3}$
}

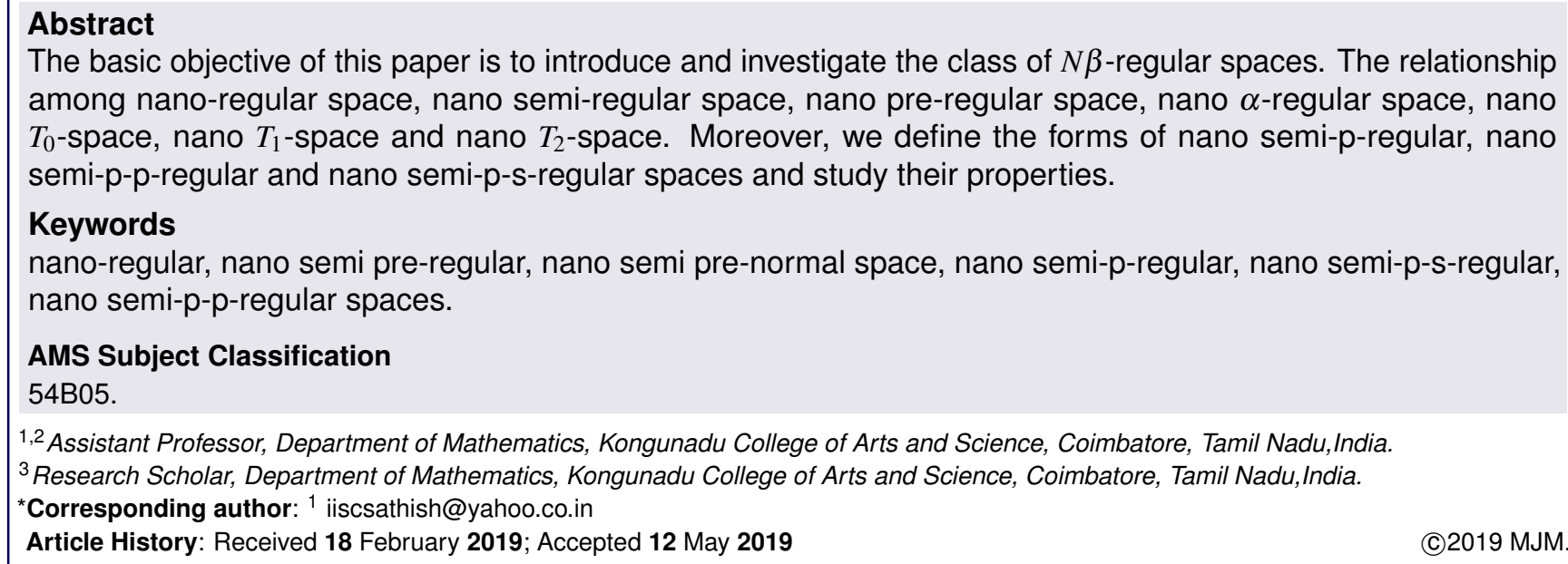
among nano-regular space, nano semi-regular space, nano pre-regular space, nano $\alpha$-regular space, nano $T_{0}$-space, nano $T_{1}$-space and nano $T_{2}$-space. Moreover, we define the forms of nano semi-p-regular, nano semi-p-p-regular and nano semi-p-s-regular spaces and study their properties.

Keywords nano-regular, nano semi pre-regular, nano semi pre-normal space, nano semi-p-regular, nano semi-p-s-regular, nano semi-p-p-regular spaces.

\section{AMS Subject Classification} 54B05.

${ }^{1,2}$ Assistant Professor, Department of Mathematics, Kongunadu College of Arts and Science, Coimbatore, Tamil Nadu,India.

${ }^{3}$ Research Scholar, Department of Mathematics, Kongunadu College of Arts and Science, Coimbatore, Tamil Nadu,India.

${ }^{\star}$ Corresponding author: ${ }^{1}$ iiscsathish@yahoo.co.in

Article History: Received 18 February 2019; Accepted 12 May 2019

(C)2019 MJM.

\section{Contents}

1 Introduction .367

2

3 Nano semi pre-regular spaces 367

4

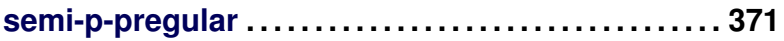

5 References 372 374

\section{Introduction}

Lellis Thivagar[1] established the notion of nano topology in terms of approximations and boundary region of a subset of an universe using an equivalence relation on it and also make known about nano-closed sets, nano-interior, nano-closure and weak form of nano open sets namely nano semi-open sets, nano pre-open, nano $\alpha$-open sets and nano semi pre-open sets. Sathishmohan et.al.[3] brings up the idea about nano neighourhoods in nano topological spaces. This motivates the author to induct and study the properties of nano semi pre-regular space and nano semi pre normal spaces in nano topological spaces.

The structure of this manuscript is as follows:

In section 2, we recall some existsing definitions and lemmas which are more important to prove our main results. In section 3, we induct and study some theorems which satisfies the conditions of $N \beta$-regular spaces.

In section 4, we introduce and examine some theorems which satisfies the conditions of nano semi-p-regular, nano semi-p-sregular, nano semi-p-p-regular spaces.

In section 5, we introduced and study some theorems which satisfies the conditions of $N \beta$-normal spaces.

\section{Preliminaries}

In this section, we recall some basic definitions and results in nano topological spaces are given, which are useful to prove the main results

Definition 2.1. [2] Let $U$ be a non-empty finite set of objects called the universe and $R$ be an equivalence relation on $U$ named as indiscernibility relation. Then $U$ is divided into disjoint equivalence classes. Elements belonging to the same equivalence class are said to be indiscernible with one another. The pair $(U, R)$ is said to be the approximation space. Let $X \subseteq U$. Then,

(i)The lower approximation of $x$ with respect to $R$ is the set of all objects, which can be for certain classified as $X$ with respect to $R$ and is denoted by $L_{R}(X)$.

$L_{R}(X)=\bigcup_{x \in U}\{R(x): R(x) \subseteq X\}$ where $R(x)$ denotes the 
equivalence class determined by $x \in U$.

(ii)The upper approximation of $x$ with respect to $R$ is the set of all objects which can be possibly classified as $X$ with respect to $R$ and is denoted by $U_{R}(X)$.

$U_{R}(X)=\bigcup_{x \in U}\{R(x): R(x) \cap X \neq \phi\}$

(iii)The boundary region of $x$ with respect to $R$ is the set of all objects which can be classified neither as $X$ nor as not-X with respect to $R$ and it is denoted by $B_{R}(X)$.

$B_{R}(X)=U_{R}(X)-L_{R}(X)$.

Definition 2.2. [2] Let $U$ be the universe, $R$ be an equivalence relation on $U$ and $\tau_{R}(X)=\left\{U, \phi, L_{R}(X), U_{R}(X), B_{R}(X)\right\}$ where $X \subseteq U$. Then $\tau_{R}(X)$ satisfies the following axioms

(i) $U$ and $\phi \in \tau_{R}(X)$.

(ii) The union of the elements of any sub-collection of $\tau_{R}(X)$ is in $\tau_{R}(X)$.

(iii) The intersection of the elements of any finite sub collection of $\tau_{R}(X)$ is in $\tau_{R}(X)$.

Then $\tau_{R}(X)$ is a topology on $U$ called the nano topology on $U$ with respect to $X$. We call $\left(U, \tau_{R}(X)\right)$ as nano topological space. The elements of $\tau_{R}(X)$ are called as nano-open sets. The complement of the nano-open sets are called nano-closed sets.

Remark 2.3. [2] If $\tau_{R}(X)$ is the nano topology on $U$ with respect to $X$, then the set $B=\left\{U, L_{R}(X), B_{R}(X)\right\}$ is the basis for $\tau_{R}(X)$.

Definition 2.4. [1] If $\left(U, \tau_{R}(X)\right)$ is a nano topological space with respect to $X$ where $X \subseteq U$ and if $A \subseteq U$, then

(i)The nano interior of $A$ is defined as the union of all nanoopen subsets of $A$ is contained in $A$ and is denoted by $\operatorname{Nint}(A)$. That is, Nint $(A)$ is the largest nano-open subset of $A$.

(ii)The nano closure of $A$ is defined as the intersection of all nano-closed sets containing $A$ and is denoted by $N \operatorname{cl}(A))$. That is, $\operatorname{Ncl}(A))$ is the smallest nano-closed set containing $A$.

Definition 2.5. [6] If $\left(U, \tau_{R}(X)\right)$ is a nano topological space with respect to $X$ where $X \subseteq U$ and if $A \subseteq U$, then

(i)The $N \beta$ interior of the set $A$ is defined as the union of all $N \beta$ open subsets contained in $A$ and is denoted by $N \beta \operatorname{Int}(A)$. $N \beta \operatorname{Int}(A)$ is the largest $N \beta$ open subset of $A$.

(ii)The $N \beta$ closure of the set $A$ is defined as the intersection of all $N \beta$ closed sets containing $A$ and is denoted by $N \beta \operatorname{cl}(A)$. $N \beta c l(A)$ is the smallest $N \beta$ closed set containing $A$.

Definition 2.6. [6] A subset $M_{x} \subset U$ is called a $N \beta$-neighbourhood $(N \beta$-nhd) of a point $x \in U$ iff there exists $a A \in N \beta O(U, X)$ such that $x \in A \subset M_{x}$ and a point $x$ is called $N \beta$-nhd point of the set $A$.

Definition 2.7. [4] A space $U$ is called nano- $T_{0}$ (or $N-T_{0}$ ) for $x, y \in U$ and $x \neq y$, there exists a nano-open set $G$ such that $x \in G$ and $y \notin G$.

Definition 2.8. [4] A space $U$ is called nano semi-T $T_{0}$ (or NS$T_{0}$ ) for $x, y \in U$ and $x \neq y$, there exists a nano semi-open set $G$ such that $x \in G$ and $y \notin G$.
Definition 2.9. [4] A space $U$ is called nano pre- $T_{0}$ (or $N P$ $\left.T_{0}\right)$ for $x, y \in U$ and $x \neq y$, there exists a nano pre-open set $G$ such that $x \in G$ and $y \notin G$.

Definition 2.10. [4] A space $U$ is called nano- $T_{1}$ (or $N-T_{1}$ ) for $x, y \in U$ and $x \neq y$, there exists a nano-open sets $G$ and $H$ such that $x \in G, y \notin G$ and $y \in H, x \notin H$.

Definition 2.11. [4] A space $U$ is called nano semi- $T_{1}$ (or $N S$ - $T_{1}$ ) for $x, y \in U$ and $x \neq y$, there exists a nano semi-open sets $G$ and $H$ such that $x \in G, y \notin G$ and $y \in H, x \notin H$.

Definition 2.12. [4] A space $U$ is called nano pre- $T_{1}$ (or $N P$ $T_{1}$ ) for $x, y \in U$ and $x \neq y$, there exists a nano pre-open sets $G$ and $H$ such that $x \in G, y \notin G$ and $y \in H, x \notin H$.

Definition 2.13. [4] A space $U$ is called nano- $T_{2}$ (or $\left.N-T_{2}\right)$ for $x, y \in U$ and $x \neq y$, there exists disjoint nano-open sets $G$ and $H$ such that $x \in G$ and $y \in H$.

Definition 2.14. [4] A space $U$ is called nano semi-T $T_{2}$ (or NS$T_{2}$ ) for $x, y \in U$ and $x \neq y$, there exists disjoint nano semi-open sets $G$ and $H$ such that $x \in G$ and $y \in H$.

Definition 2.15. [4] A space $U$ is called nano pre- $T_{2}$ (or $N P$ $T_{2}$ ) for $x, y \in U$ and $x \neq y$, there exists disjoint nano pre-open sets $G$ and $H$ such that $x \in G$ and $y \in H$.

Definition 2.16. [7] A space $U$ is called nano $\alpha-T_{0}$ (or $N \beta$ $T_{0}$ ) for $x, y \in U$ and $x \neq y$, there exists a nano $\alpha$-open set $G$ such that $x \in G$ and $y \notin G$.

Definition 2.17. [7] A space $U$ is called $N \beta-T_{0}\left(\operatorname{or} N \beta-T_{0}\right)$ for $x, y \in U$ and $x \neq y$, there exists a $N \beta$-open set $G$ such that $x \in G$ and $y \notin G$.

Definition 2.18. [7] A space $U$ is called nano $\alpha-T_{1}$ (or $N \alpha$ $\left.T_{1}\right)$ for $x, y \in U$ and $x \neq y$, there exists a $N \alpha$-open sets $G$ and $H$ such that $x \in G, y \notin G$ and $y \in H, x \notin H$.

Definition 2.19. [7] A space $U$ is called $N \beta-T_{1}$ (or $\left.N \beta-T_{1}\right)$ for $x, y \in U$ and $x \neq y$, there exists a $N \beta$-open sets $G$ and $H$ such that $x \in G, y \notin G$ and $y \in H, x \notin H$.

Definition 2.20. [7] A space $U$ is called nano $\alpha-T_{2}$ (or $N \alpha$ $T_{2}$ ) for $x, y \in U$ and $x \neq y$, there exists disjoint $N \alpha$-open sets $G$ and $H$ such that $x \in G$ and $y \in H$.

Definition 2.21. [7] A space $U$ is called nano semipre- $T_{2}$ (or $N \beta-T_{2}$ ) for $x, y \in U$ and $x \neq y$, there exists disjoint $N \beta$-open sets $G$ and $H$ such that $x \in G$ and $y \in H$.

Definition 2.22. [5] A subset A of a nano topological space $\left(U, \tau_{R}(X)\right)$ is nano-regular open in $U$, if $N \operatorname{Int}(N c l(A))=A$.

Definition 2.23. [5] A space $U$ is said to be nano semiregular if for each nano semi-closed set $F$ and for each $x \in U-F$ there exists nano-open sets $G$ and $H$ such that $F \subset G$ and $x \in H$. It is denoted by NS-regular space. 
Definition 2.24. [5] A space $U$ is said to be nano pre-regular iffor each nano pre-closed set $F$ and for each $x \in U-F$ there exists nano-open sets $G$ and $H$ such that $F \subset G$ and $x \in H$. It is denoted by NP-regular space.

Lemma 2.25. [7] Let $A$ and $Y$ be subsets of $U$ such that $A \subset Y$ and $Y$ is $N \beta$-open then $A$ is $N \beta$-open subset of $Y$ iff $A$ is $N \beta$-open subset of $U$.

Lemma 2.26. [7] If $A$ is $N \beta$-open in $U$ and $Y \in \alpha(U), A \cap Y$ is $N \beta$-open subset of $U$.

\section{Nano semi pre-regular spaces}

In this section, we introduce $N \beta$-regular spaces by using $N \beta$ open sets and study some of its properties.

Definition 3.1. A space $U$ is said to be nano semi pre-regular if for each $N \beta$-closed set $F$ and for each $x \in U-F$ there exists nano-open sets $G$ and $H$ such that $F \subset G$ and $x \in H$ and it is denoted by $N \beta$-regular space.

Theorem 3.2. : Every nano-regular space is $N \beta$ (resp. NS, NP)-regular space.

Proof. Let $U$ be nano-regular and $F$ be a nano-closed set not containing $x$ implies $F$ be $N \beta$ (resp. $N S, N P$ )-closed set not containing $x$. As $U$ is $N \beta$ (resp. $N S, N P$ )-regular there exists disjoint $N \beta$ (resp. $N S, N P$ )-open sets $G$ and $H$ such that $x \in H$ and $F \subset G$. Therefore $U$ is $N \beta$ (resp. $N S, N P$ )-regular. Converse of this theorem is need not be true in general.

Example 3.3. Let $U=\{a, b, c, d\}, U / R=\{\{a\},\{b, d\},\{c\}\}$ $X=\{a, b\}$ and $\tau_{R}(X)=\{U, \phi,\{a\},\{a, b, d\},\{b, d\}\}$ be $a$ nano topology on $U$, we have

$\operatorname{NSR}(U, X)=\{U, \phi,\{a\},\{a, c\},\{b, d\},\{b, c, d\}\}$

$N P R(U, X)=\{U, \phi,\{b\},\{d\},\{a, b, c\},\{a, c, d\}\}$

$N \alpha R(U, X)=\{U, \phi\}$ and $N \beta R(U, X)=\{U, \phi,\{a\},\{b\},\{d\}$, $\{a, b\},\{a, c\},\{a, d\},\{b, c\},\{b, d\},\{c, d\},\{a, b, c\},\{a, c, d\}$, $\{b, c, d\}\}$.

1. Let $x=\{b, c, d\}$, be a $N \beta($ resp. NS)-regular space but not nano-regular space.

2. Let $x=\{a, c, d\}$, be a NP-regular space but not nanoregular space.

Theorem 3.4. : Every nano $\alpha$-regular space is $N \beta$ (resp. $N S$, NP)-regular space.

Proof. Let $U$ be nano $\alpha$-regular and $F$ be a nano $\alpha$-closed set not containing $x$ implies $F$ be $N \beta$ (resp. $N S, N P$ )-closed set not containing $x$. As $U$ is $N \beta$ (resp. $N S, N P$ )-regular there exists disjoint $N \beta$ (resp. $N S, N P$ )-open sets $G$ and $H$ such that $x \in H$ and $F \subset G$. Therefore $U$ is $N \beta$ (resp. $N S, N P$ )-regular. Converse of the theorem is need not be true in general.

Example 3.5. From the Example 3.3,
1. Let $x=\{b, c, d\}$, be a $N \beta$ (resp. NS)-regular space but not nano $\alpha$-regular space.

2. Let $x=\{a, c, d\}$, be a NP-regular space but not nano $\alpha$-regular space.

Theorem 3.6. : Every nano semi-regular space is $N \beta$-regular space.

Proof. Let $U$ be nano semi-regular and $F$ be a nano semiclosed set not containing $x$ implies $F$ be $N \beta$-closed set not containing $x$. As $U$ is $N \beta$-regular there exists disjoint $N \beta$ open sets $G$ and $H$ such that $x \in H$ and $F \subset G$. Therefore $U$ is $N \beta$-regular.

Converse of the theorem is need not be true in general.

Example 3.7. From the Example 3.3, Let $x=\{a, c, d\}$, be $a$ $N \beta$-regular space but not $N S$-regular space.

Theorem 3.8. : Every nano pre-regular space is $N \beta$-regular space.

Proof. Let $U$ be nano pre-regular and $F$ be a nano pre-closed set not containing $x$ implies $F$ be $N \beta$-closed set not containing $x$. As $U$ is $N \beta$-regular there exists disjoint $N \beta$-open sets $G$ and $H$ such that $x \in H$ and $F \subset G$. Therefore $U$ is $N \beta$-regular. Converse of the theorem is need not be true in general.

Example 3.9. From the Example 3.3, Let $x=\{b, c, d\}$, be $a$ $N \beta$-regular space but not $N P$-regular space.

Theorem 3.10. Every nano- $T_{1}$ and nano-regular space is nano- $T_{0}$ space.

Proof. Let $U$ be nano- $T_{1}$ and nano-regular. As $U$ is nano- $T_{1}$, every singleton set $\{x\}$ is nano-closed for all $x \in U$ and $U$ being nano-regular and $\{\mathrm{x}\}$ is a nano-closed subset of $U$ and $y$ be any point of $U-\{x\}$ then $x \neq y$. By criteria for nanoregularity there exists two disjoint nano-open sets $G$ and $H$ such that $x \subset G$ and $y \notin H \Rightarrow x \in G$ and $y \notin H \Rightarrow\left(U, \tau_{R}(X)\right)$ is nano- $T_{0}$ space.

Theorem 3.11. Every $N S-T_{1}$ and $N S$-regular space is $N S-T_{0}$ space.

Proof. Let $U$ be $N S-T_{1}$ and $N S$-regular. As $U$ is $N S-T_{1}$, every singleton set $\{x\}$ is $N S$-closed for all $x \in U$ and $U$ being $N S$ regular and $\{\mathrm{x}\}$ is a $N S$-closed subset of $U$ and $y$ be any point of $U-\{x\}$ then $x \neq y$. By criteria for $N S$-regularity there exists two disjoint $N S$-open sets $G$ and $H$ such that $x \subset G$ and $y \notin H \Rightarrow x \in G$ and $y \notin H \Rightarrow\left(U, \tau_{R}(X)\right)$ is $N S$ - $T_{0}$ space.

Theorem 3.12. Every $N P-T_{1}$ and $N P$-regular space is $N P-T_{0}$ space.

Proof. Let $U$ be $N P-T_{1}$ and $N P$-regular. As $U$ is $N P-T_{1}$, every singleton set $\{x\}$ is $N P$-closed for all $x \in U$ and $U$ being $N P$ regular and $\{\mathrm{x}\}$ is a $N P$-closed subset of $U$ and $y$ be any point of $U-\{x\}$ then $x \neq y$. By criteria for $N P$-regularity there exists two disjoint $N P$-open sets $G$ and $H$ such that $x \subset G$ and $y \notin H \Rightarrow x \in G$ and $y \notin H \Rightarrow\left(U, \tau_{R}(X)\right)$ is $N P-T_{0}$ space. 
Remark 3.13. The converse of the above Theorems 3.10, 3.11 and 3.12 need not be true in general as shown in the following example.

Example 3.14. Let $U=\{a, b, c, d\}, U / R_{1}=\{\{a\},\{b, d\},\{c\}\}$ $X_{G}=\{a, b\}$ and $\tau_{R}\left(X_{G}\right)=\{U, \phi,\{a\},\{a, b, d\},\{b, d\}\}$ be $a$ nano topology on $U$, we have

$\operatorname{NSO}\left(U, \tau_{R}\left(X_{G}\right)\right)=\{U, \phi,\{a\},\{a, c\},\{b, d\},\{a, b, d\},\{b, c, d\}\}$ $N P O\left(U, \tau_{R}\left(X_{G}\right)\right)=\{U, \phi,\{a\},\{b\},\{d\},\{a, b\},\{a, d\},\{b, d\}$, $\{a, b, c\},\{a, b, d\},\{a, c, d\}$,

$N \alpha O\left(U, \tau_{R}\left(X_{G}\right)\right)=\{U, \phi,\{a\},\{a, b, d\},\{b, d\}\}$

$N \beta O\left(U, \tau_{R}\left(X_{G}\right)\right)=\{U, \phi,\{a\},\{b\},\{d\},\{a, b\},\{a, c\},\{a, d\}$, $\{b, c\},\{b, d\},\{c, d\},\{a, b, c\},\{a, b, d\},\{a, c, d\},\{b, c, d\}\}$

$\operatorname{NSR}\left(U, \tau_{R}\left(X_{G}\right)\right)=\{U, \phi,\{a\},\{a, c\},\{b, d\},\{b, c, d\}\}$

$\operatorname{NPR}\left(U, \tau_{R}\left(X_{G}\right)\right)=\{U, \phi,\{b\},\{d\},\{a, b, c\},\{a, c, d\}\}$

$N \alpha R\left(U, \tau_{R}\left(X_{G}\right)\right)=\{U, \phi$,$\} and$

$N \beta R\left(U, \tau_{R}\left(X_{G}\right)\right)=\{U, \phi,\{a\},\{b\},\{d\},\{a, b\},\{a, c\},\{a, d\}$, $\{b, c\},\{b, d\},\{c, d\},\{a, b, c\},\{a, c, d\},\{b, c, d\}\}$.

$U / R_{2}=\{\{a, b\},\{c\},\{d\}\} X_{H}=\{b, c\}$ and

$\tau_{R}\left(X_{H}\right)=\{U, \phi,\{c\},\{a, c, d\},\{a, d\}\}$. we have

$N S O\left(U, \tau_{R}\left(X_{H}\right)\right)=\{U, \phi,\{c\},\{a, d\},\{b, c\},\{a, b, d\},\{a, c, d\}\}$ $N P O\left(U, \tau_{R}\left(X_{H}\right)\right)=\{U, \phi,\{a\},\{c\},\{d\},\{a, c\},\{a, d\},\{b, d\}$, $\{a, b, c\},\{a, c, d\}\}$

$N \alpha O\left(U, \tau_{R}\left(X_{H}\right)\right)=\{U, \phi,\{c\},\{a, c, d\},\{a, d\}\}$

$N \beta O\left(U, \tau_{R}\left(X_{H}\right)\right)=\{U, \phi,\{b\},\{c\},\{d\},\{a, b\},\{a, c\},\{a, d\}$, $\{b, c\},\{b, d\},\{c, d\},\{a, b, c\},\{a, b, d\},\{a, c, d\},\{b, c, d\}\}$

$\operatorname{NSR}\left(U, \tau_{R}\left(X_{H}\right)\right)=\{U, \phi,\{c\},\{a, d\},\{b, c\},\{a, b, d\}\}$

$N P R\left(U, \tau_{R}\left(X_{H}\right)\right)=\{U, \phi,\{a\},\{d\},\{b, d\},\{a, b, c\},\{a, c, d\}$, $\{b, c, d\}\}$

$N \alpha R\left(U, \tau_{R}\left(X_{H}\right)\right)=\{U, \phi\}$ and

$N \beta R\left(U, \tau_{R}\left(X_{H}\right)\right)=\{U, \phi,\{b\},\{c\},\{d\},\{a, b\},\{a, c\},\{a, d\}$, $\{b, c\},\{b, d\},\{c, d\},\{a, b, c\},\{a, b, d\},\{a, c, d\}\}$

1. Let $x=\{a\}$ and $y=\{b\}$ is nano- $T_{0}$ space but not nano$T_{1}$ (resp. nano-regular space).

2. Let $x=\{a, b, d\}$ and $y=\{b\}$ is $N S-T_{0}$ space but not nano- $T_{1}$ (resp. NS-regular space).

3. Let $x=\{a\}$ and $y=\{b\}$ is NP- $T_{0}$ space but not nano- $T_{1}$ (resp. NP-regular space).

Theorem 3.15. Every $N S-T_{1}$ and $N S$-regular space is $N S-T_{2}$ space.

Proof. Let $U$ be $N S-T_{1}$ and $N S$-regular. As $U$ is $N S$ - $T_{1}$, every singleton set $\{x\}$ is $N S$-closed for all $x \in U$ and $U$ being $N S$ regular and $\{\mathrm{x}\}$ is a $N S$-closed subset of $U$ and $y$ be any point of $U-\{x\}$ then $x \neq y$. By criteria for $N S$-regularity there exists two disjoint $N S$-open sets $G$ and $H$ such that $\{x\} \subset G$ and $y \in H \Rightarrow x \in G$ and $y \in H . \Rightarrow\left(U, \tau_{R}(X)\right)$ is $N S-T_{2}$.

Theorem 3.16. Every $N P-T_{1}$ and $N P$-regular space is $N P-T_{2}$ space.

Proof. Let $U$ be $N P-T_{1}$ and $N P$-regular. As $U$ is $N P-T_{1}$, every singleton set $\{x\}$ is $N P$-closed for all $x \in U$ and $U$ being $N P$ regular and $\{\mathrm{x}\}$ is a $N P$-closed subset of $U$ and $y$ be any point of $U-\{x\}$ then $x \neq y$. By criteria for $N P$-regularity there exists two disjoint $N P$-open sets $G$ and $H$ such that $\{x\} \subset G$ and $y \in H \Rightarrow x \in G$ and $y \in H . \Rightarrow\left(U, \tau_{R}(X)\right)$ is $N P-T_{2}$.

Theorem 3.17. Every $N S-T_{1}$ and $N S$-regular space is $N P-T_{2}$ space.

Proof. Let $U$ be $N S-T_{1}$ and $N S$-regular. As $U$ is $N S-T_{1}$, every singleton set $\{x\}$ is $N S$-closed for all $x \in U$ and $U$ being $N S$-regular and $\{\mathrm{x}\}$ is a $N S$-closed subset of $U$ and $y$ be any point of $U-\{x\}$ then $x \neq y$. By criteria for $N P$-regularity there exists two disjoint $N P$-open sets $G$ and $H$ such that $\{x\} \subset G$ and $y \in H \Rightarrow x \in G$ and $y \in H \Rightarrow\left(U, \tau_{R}(X)\right)$ is $N P-T_{2}$ space.

Theorem 3.18. Every $N S-T_{1}$ and $N S$-regular space is $N \beta-T_{2}$ space.

Proof. Let $U$ be $N S-T_{1}$ and $N S$-regular. As $U$ is $N S-T_{1}$, every singleton set $\{x\}$ is $N S$-closed for all $x \in U$ and $U$ being $N S$-regular and $\{\mathrm{x}\}$ is a $N S$-closed subset of $U$ and $y$ be any point of $U-\{x\}$ then $x \neq y$. By criteria for $N \beta$-regularity there exists two disjoint $N \beta$-open sets $G$ and $H$ such that $\{x\} \subset G$ and $y \in H \Rightarrow x \in G$ and $y \in H \Rightarrow\left(U, \tau_{R}(X)\right)$ is $N \beta-T_{2}$ space.

Remark 3.19. $N S-T_{2}$ (resp. $\left.N P-T_{2}, N \beta-T_{2}\right)$ and $N S$ (resp. $N P, N \beta)$-regular space is $N \beta-T_{0}$ space.

Remark 3.20. The converse of the above Theorems 3.15, 3.16, 3.17 and 3.18 are need not be true in general as shown in the following example.

Example 3.21. From the Example 3.14

1. Let $x=\{a\}$ and $y=\{b\}$ is nano- $T_{2}$ space but not nanoregular space.

2. Let $x=\{a, b, d\}$ and $y=\{b\}$ is NS-T $T_{2}$ space but not NS-regular space.

3. Let $x=\{a\}$ and $y=\{b\}$ is NP-T space but not $N P$ regular space.

Theorem 3.22. : For a nano topological space $U$ the following statements are equivalent:

1. $U$ is $N \beta$-regular

2. For each $x \in U$ and for each $N \beta$-open set $G$ containing $x$ there exists a $N \beta$-open set $H$ containing $x$ such that $x \in H \subset N \beta \operatorname{cl}(H) \subset G$.

3. For each $N \beta$-closed set $F$ of $U, \cap\{N \beta c l(H) / F \subset H$ and $H \in N \beta O(U)\}=F$

4. For each non-empty subset $A$ of $U$ and each $G \in N \beta O(U)$ if $A \cap G \neq \phi$ then there exists $H \in N \beta O(U)$ such that $A \cap H \neq \phi$ and $N \beta c l(H) \subset G$ 
5. For each non-empty subset $A$ of $U$ and each $F \in N \beta F(U)$ if $A \cap F=\phi$ then there exists $H, W \in N \beta O(U)$ such that $A \cap H \neq \phi, F \subset W$ and $H \cap W=\phi$.

Proof. (1) $\Rightarrow(2)$ : Let $U$ be $N \beta$-regular space. Let $x \in U$ and $G$ be $N \beta$-open set containing $x \Rightarrow U-G$ is $N \beta$-closed such that $x \in U-G$. Therefore by a) there exists two $N \beta$-open sets $H$ and $W$ such that $x \in H$ and $U-G \subset W \Rightarrow U-W \subset G$. Since $H \cap W=\phi \Rightarrow N \beta c l(H) \cap W=\phi . \quad \Rightarrow N \beta c l(H) \subset$ $U-W \subset G$. Therefore $x \in H \subset N \beta c l(H) \subset G$.

(2) $\Rightarrow$ (3) : Let $F$ be a $N \beta$-closed subset of $U$ and $x \notin$ $F$ then $U-F$ is $N \beta$-open set containing $x$. By b) there exists $N \beta$-open set $G$ such that $x \in G \subset N \beta c l(G) \subset U-$ $F \Rightarrow F \subset U-N \beta c l(G) \subset U-G$ i.e $F \subset H \subset U-G$ where $H=U-N \beta c l(G) \in N \beta O(U)$ and $x \notin H$ that implies $x \notin$ $N \beta \operatorname{cl}(H) \Rightarrow x \notin \cap\{N \beta c l(H) / F \subset H \in N \beta O(U)\}$. Hence $\cap\{N \beta c l(H) / F \subset H \in N \beta O(U)\}=F$.

(3) $\Rightarrow$ (4) : A be a subset of $U$ and $G \in N \beta O(U)$ such that $A \cap G \neq \phi$, that implies there exists $x_{o} \in U$ such that $x_{0} \in$ $A \cap G$. Therefore $U-G$ is $N \beta$-closed set not containing $x_{0} \Rightarrow$ $x_{0} \notin N \beta c l(U-G)$. By c) there exists $W \in N \beta O(U)$ such that $U-G \subset W \Rightarrow x_{0} \notin N \beta c l(W)$. Put $H=U-N \beta c l(W)$ then $H$ is $N \beta$-open set containing $x_{0} . \Rightarrow A \cap H \neq \phi$ and $N \beta c l(H) \subset(N \beta c l(U)-N \beta c l(W)) \subset N \beta c l(U-W)$. Therefore $N \beta \operatorname{cl}(H) \subset N \beta c l(U-W) \subset G$.

(4) $\Rightarrow(5)$ : Let $A$ be a nonempty subset of $U$ and $F$ be $N \beta$ closed set such that $A \cap F=\phi$. Then $U-F$ is $N \beta$-open in $U$ and $A \cap(U-F) \neq \phi$. Therefore by d) there exists $H \in N \beta O(U)$ such that $A \cap H \neq \phi$ and $N \beta c l(H) \subset U-F$. Put $W=U-N \beta \operatorname{cl}(H)$ then $W \in N \beta O(U)$ such that $F \subset W$ and $W \cap H=\phi$.

$(5) \Rightarrow(1)$ : Let $x \in U$ be arbitrary and $F$ be $N \beta$-closed set not containing $x$. Let $A$ be a nonempty nano-open set containing $x$ then by e) there exists disjoint $N \beta$-open sets $H$ and $W$ such that $F \subset W$ and $A \cap H \neq \phi \Rightarrow x \in H$. Thus $U$ is $N \beta$-regular.

Theorem 3.23. : A space $U$ is $N \beta$-regular iff for each $N \beta$ open set $W$ of $U$ and each $x \in G$ there exists $N \beta$-regular set $H$ such that $x \in H \subset G$.

Proof. Let $U$ be $N \beta$-regular and $G$ be an arbitrary $N \beta$-open then by Theorem 3.22 there exists $N \beta$-open $W$ such that $x \in$ $W \subset N \beta c l(W) \subset G$. By Lemma 2.25 as $W$ is $N \beta$-open subset of $U, N \beta c l(W)$ is $N \beta$-regular and let $H=N \beta c l(W)$ such that $x \in W \subset H \subset G \Rightarrow x \in H \subset G$ where $H$ is $N \beta$-regular.

Conversely: For each $G \in N \beta O(U)$ and for each $x \in G$ there exists $H \in N \operatorname{SPR}\left(U, \tau_{R}(X)\right)$ such that $x \in H \subset G$. As $H$ is $N \beta$-closed, $H=N \beta c l(H)$. Therefore $x \in H=N \beta \operatorname{cl}(H) \subset G$ i.e $x \in H \subset N \beta c l(H) \subset G$. $\Rightarrow U$ is $N \beta$-regular.

Theorem 3.24. A topological space $\left(U, \tau_{R}(X)\right)$ is $N \beta$-regular iff each $N \beta$-open neighbourhood of an arbitrary element $x \in$ $U$ contains the $N \beta$-closure of another $N \beta$-neighbourhood of $x$.

Proof. Let $x$ be any point of $N \beta$-regular space $U$ and $\mathscr{N}$ be an arbitrary $N \beta$-open neighbourhood of $x$. Hence $U-\mathscr{N}$ is semi pre-closed set such that $x \notin U-\mathscr{N}$. From the criteria of semi pre-regularity there exists two semi pre-open sets $M$ and $L$ such that $U-\mathscr{N} \subset L, x \in M$ and $L \cap M=\phi$. Now $L \cap M=\phi \Rightarrow M \subset U-L$. Therefore $N \beta c l(M) \subset$ $N \beta c l(U-L)=U-L$.

i.e $N \beta c l(M) \subset U-L \ldots \ldots . . .(1)$ Again $U-\mathscr{N} \subset L \Rightarrow U-L \subset$

$\mathscr{N}$........(2) From (1) and (2) we have $N \beta c l(M) \subset \mathscr{N}$

Since $M$ is $N \beta$-open set containing $x \Rightarrow$ it is $N \beta$-neighbourhood of $x$ and thus from (3) we conclude that each $N \beta$-open neighbourhood $\mathscr{N}$ of an arbitrary element $x$ contains $N \beta c l(M)$. i.e $N \beta$-closure of another neighbourhood of $x$.

\section{Nano semi-p-regular, Nano semi-p-s-regular and Nano semi-p-pregular}

In this section we introduce the nano semi-p-regular, nano semi-p-s-regular and nano semi-p-p-regular spaces and study the conditions which satisfies our definitions.

Definition 4.1. A space $U$ is said to be nano semi-p-regular if for each $N \beta$-closed set $F$ and for each $x \in U-F$ there exists disjoint nano open sets $G$ and $H$ such that $F \subset G$ and $x \in H$.

Definition 4.2. A space $U$ is said to be nano semi-p-s-regular if for each $N \beta$-closed set $F$ and for each $x \in U-F$ there exists disjoint nano semi open sets $G$ and $H$ such that $F \subset G$ and $x \in H$.

Definition 4.3. A space $U$ is said to be nano semi-p-p-regular iffor each $N \beta$-closed set $F$ and for each $x \in U-F$ there exists disjoint nano pre open sets $G$ and $H$ such that $F \subset G$ and $x \in H$.

Theorem 4.4. Every nano semi-p-regular is $N \beta$-regular space.

Proof. Let $U$ be nano semi-p-regular and $F$ be any $N \beta$-closed set and $x \in F$. Then there exists disjoint nano open sets $G$ and $H$ such that $x \in G$ and $F \subset H$. Since every nano open set is $N \beta$-open and hence $G$ and $H$ are $N \beta$-open sets such that $x \in G$ and $F \subset H$. This shows that $U$ is $N \beta$-regular.

In the same way one can prove every nano semi-p-sregular is $N \beta$-regular space and nano semi-p-p-regular is $N \beta$ regular space.

Example 4.5. Let $U=\{a, b, c, d\}, U / R_{1}=\{\{a\},\{b, d\},\{c\}\}$ $X=\{a, b\}$ and $\tau_{R}(X)=\{U, \phi,\{a\},\{a, b, d\},\{b, d\}\}$ be $a$ nano topology on $U$, we have

$\operatorname{NSO}\left(U, \tau_{R}(X)\right)=\{U, \phi,\{a\},\{a, c\},\{b, d\},\{a, b, d\},\{b, c, d\}\}$ $N P O\left(U, \tau_{R}(X)\right)=\{U, \phi,\{a\},\{b\},\{d\},\{a, b\},\{a, d\},\{b, d\}$, $\{a, b, c\},\{a, b, d\},\{a, c, d\}$, $N \alpha O\left(U, \tau_{R}(X)\right)=\{U, \phi,\{a\},\{a, b, d\},\{b, d\}\}$ $N \beta O\left(U, \tau_{R}(X)\right)=\{U, \phi,\{a\},\{b\},\{d\},\{a, b\},\{a, c\},\{a, d\}$, $\{b, c\},\{b, d\},\{c, d\},\{a, b, c\},\{a, b, d\},\{a, c, d\},\{b, c, d\}\}$ $\operatorname{NSR}\left(U, \tau_{R}(X)\right)=\{U, \phi,\{a\},\{a, c\},\{b, d\},\{b, c, d\}\}$ $N P R\left(U, \tau_{R}(X)\right)=\{U, \phi,\{b\},\{d\},\{a, b, c\},\{a, c, d\}\}$ 
$N \alpha R\left(U, \tau_{R}(X)\right)=\{U, \phi$,$\} and$

$N \beta R\left(U, \tau_{R}(X)\right)=\{U, \phi,\{a\},\{b\},\{d\},\{a, b\},\{a, c\},\{a, d\}$, $\{b, c\},\{b, d\},\{c, d\},\{a, b, c\},\{a, c, d\},\{b, c, d\}\}$.

Let $x=\{c, d\}$ is $N \beta$-regular but not nano semi-p-s-regular (resp. semi-p-p-regular)

Theorem 4.6. For a nano topological space $U$ the following statements are equivalent:

(a) $U$ is nano semi-p-regular

(b) For each $x \in U$ and for each $N \beta$-open set $G$ of $U \ni x$ if open set $H$ such that $x \in H \subset N \beta c l(H) \subset G$.

(c) For each $N \beta$-closed set $F$ of $U, \cap\{N c l(H) / F \subset H \in$ $\left.\tau_{R}(X)\right\}=F$

(d) For each nonempty subset $A$ of $U$ and each $G \in N \beta O(U)$ if $A \cap G \neq \phi$ then there exists open set $H$ such that $A \cap H \neq \phi$ and $\mathrm{Ncl}(\mathrm{H}) \subset \mathrm{G}$

(e) For each nonempty subset A of $U$ and each closed set $F$ of $U$ such that $A \cap F=\phi$, there exits, $H, W \in N \beta O(U)$, such that $A \cap H \neq \phi, F \subset W$ and $H \cap W=\phi$.

Proof. (a) $\Rightarrow$ (b) : Let $x \in U$ and $G$ be $N \beta$-open set of $U$ containing $x \Rightarrow U-G$ is $N \beta$-closed set not containing $x$. As $U$ is nano semi-p-regular there exists disjoint nano open sets $H$ and $W$ such that $x \in H$ and $U-G \subset W \Rightarrow U-W \in G$. As $H \cap W=\phi, N c l(H) \cap W=\phi \Rightarrow N c l(H) \subset U-W \subset G$. Hence $x \in H \subset N \operatorname{cl}(H) \subset G$.

(b) $\Rightarrow$ (c) : Let $F$ be a $N \beta$-closed subset of $U$ and $x \notin F$ then $U-F$ is $N \beta$-open set containing $x$. By b) there exists nano open set $G$ such that $x \in G \subset \operatorname{Ncl}(G) \subset U-F \Rightarrow F \subset$ $U-\operatorname{Ncl}(G) \subset U-G$. i.e $F \subset H \subset U-G$ where $H=U-$ $N c l(G) \in x$ and $x \neq H \Rightarrow x \notin N c l(H) \Rightarrow F \subset \cap\{N c l(H) / F \subset$ $\left.H \in \tau_{R}(X)\right\}$. Hence $\cap\left\{N \operatorname{cl}(H) / F \subset H \in \tau_{R}(X)\right\}=F$

(c) $\Rightarrow$ (d): $A$ be a subset of $U$ and $G \in N \beta O(U)$ such that $A \cap G \neq \phi$ which implies that there exists $x_{0} \in U$ such that $x_{0} \in A \cap G$. Therefore $U-G$ is nano semi-pre closed set not containing $x_{0} \Rightarrow x_{0} \notin N \operatorname{cl}(U-G)$. By c) there exists nano open set $W$ such that $U-G \subset W \Rightarrow x_{0} \notin N c l(W)$. Put $H=U-N c l(W)$ then $H$ is nano open set containing $x_{0}$ hence $x_{0} \in A \cap H$, This implies $A \cap H \neq \phi$ and $N \operatorname{cl}(H)=N \operatorname{Nol}(U-$ $N \operatorname{cl}(W)) \subset N \operatorname{Nl}(U-W)=U-W$. Therefore $N c l(H) \subset(U-$ $W) \subset G$. Thus for each subset $A$ of $U$ for each $N \beta$-open set $G$ such that $A \cap G \neq \phi$ there exists nano open set $H$ such that $A \cap H \neq \phi$ and $\mathrm{Ncl}(H) \subset G$.

(d) $\Rightarrow$ (e): Let $A$ be a nonempty subset of $U$ and $F$ be $N \beta$ closed set such that $A \cap F=\phi$. Then $U-F$ is $N \beta$-open in $U$ and $A \cap(U-F) \neq \phi$. Therefore from d) there exists nano open set $H$ such that $A \cap H \neq \phi$ and $N c l(H) \subset U-F$. Put $W=U-N \operatorname{cl}(H)$ then $W$ is nano closed set such that $F \subset W$ and $W \cap H=\phi$.

(e) $\Rightarrow$ (a): Let $x \in U$ be arbitrary and $F$ be $N \beta$-closed set not containing $x$. Then $U-F$ be $N \beta$-open set containing $x$. Clearly $U-(U-F)=\phi$ then by e) there exists disjoint nano-open sets $H$ and $W$ such that $F \subset W$ and $x \in H$ and $H \cap W=\phi$. Thus $U$ is nano semi-p-regular.

Theorem 4.7. A space $U$ is nano semi-p-p-regular iff for each
$N \beta$-open set $G$ and for each $x \in G$ there exists nano closed set $H$ such that $x \in H \subset G$.

Proof. Let $U$ be nano semi-p-regular and $G$ be any $N \beta$-open set of $U$ such that $x \in G$. Then there exists nano open set $W$ such that $x \in W \subset N c l(W) \subset G$ i.e $x \in H \subset G$ where $\operatorname{Ncl}(W)=H$ is nano closed set.

Theorem 4.8. In a nano topological space $U$ following statements are equivalent; (a) space $U$ is nano semi-p-s-regular (b) for each $N \beta$-open set $G$ of $U$ containing $x$ there exists nano semi-open set $H$ such that $x \in H \subset N \operatorname{scl}(H) \subset G$ (c) for each $N \beta$-open set $G$ of $U$ containing $x$ there exists nano semi-closed set $W$ such that $x \in W \subset G$

Proof. (a) $\Rightarrow$ (b) : Let $x \in U$ and $G$ be $N \beta$-open set of $U$ containing $x \Rightarrow U-G$ is $N \beta$-closed set not containing $x$. As $U$ is nano semi-p-s-regular there exists disjoint nano semi-open sets $H$ and $W$ such that $x \in H$ and $U-G \subset W \Rightarrow U-W \subset G$. As $H \cap W=\phi \Rightarrow N \operatorname{scl}(H) \cap W=\phi \Rightarrow N \operatorname{scl}(H) \subset U-W \subset G$. Hence $x \in H \subset N \operatorname{scl}(H) \subset G$.

(b) $\Rightarrow$ (c): From b) for $N \beta$-open set $G$ of $U$ containing $x$ there exists nano semi-open set $H$ such that $x \in H \subset N \operatorname{scl}(H) \subset G$ i.e $x \in W \subset H$ where $\operatorname{Nscl}(H)=W$ is nano semi-closed set. Therefore for each $N \beta$-open set $G$ of $U$ containing $x$ there exists nano semi-closed set $W$ such that $x \in W \subset G$.

(c) $\Rightarrow$ (a): Let for each $x \in U, F$ be $N \beta$-closed set not containing $x$, therefore $U-F$ is $N \beta$-open set containing $x$ hence from b) there exists nano-semi open set $H$ such that $x \in H \subset$ $N \operatorname{Ncl}(H) \subset U-F$. Let $G=U-N \operatorname{scl}(H)$ then $G$ is nano semiopen set such that $F \subset G, x \in H$ and $G \cap H=\phi$. Thus there exists disjoint nano semi-open sets $G$ and $H$ such that $x \in H$ and $F \subset G$. Therefore $U$ is nano semi-p-s-regular.

\section{Nano semi pre-normal space}

Definition 5.1. A nano topological space $\left(U, \tau_{R}(X)\right)$ is called nano-normal space if corresponding to every pair of disjoint nano-closed sets $A$ and $B$ of $U$ their exists nano-open sets $G$ and $H$ such that $A \subset G$ and $B \subset H$ and $G \cap H=\phi$. It is denoted by $N$-normal space.

Definition 5.2. A nano topological space $\left(U, \tau_{R}(X)\right)$ is called nano semi-normal space if corresponding to every pair of disjoint nano semi-closed sets $A$ and $B$ of $U$ their exists nano semi-open sets $G$ and $H$ such that $A \subset G$ and $B \subset H$ and $G \cap H=\phi$. It is denoted by NS-normal space.

Definition 5.3. A nano topological space $\left(U, \tau_{R}(X)\right)$ is called nano pre-normal space if corresponding to every pair of disjoint nano pre-closed sets $A$ and $B$ of $U$ their exists nano preopen sets $G$ and $H$ such that $A \subset G$ and $B \subset H$ and $G \cap H=\phi$. It is denoted by NP-normal space.

Definition 5.4. A nano topological space $\left(U, \tau_{R}(X)\right)$ is called nano semi pre-normal space if corresponding to every pair of disjoint $N \beta$-closed sets $A$ and $B$ of $U$ their exists $N \beta$-open sets $G$ and $H$ such that $A \subset G$ and $B \subset H$ and $G \cap H=\phi$. It is denoted by $N \beta$-normal space. 
Theorem 5.5. Every nano (resp. NS, NP) normal space is $N \beta$-normal space.

Proof. Let $U$ be nano (resp. NS, NP)-normal space and $A$ and $B$ be arbitrary pair of disjoint nano (resp. $N S, N P$ )-closed sets. As every nano (resp. $N S, N P$ )-closed set is $N \beta$-closed set, $A$ and $B$ are $N \beta$-closed sets and $U$ is $N \beta$-normal, therefore there exists disjoint $N \beta$-open sets $G$ and $H$ such that $A \subset G$ and $B \subset H$. Thus for every pair of disjoint nano (resp. $N S$, $N P$ )-closed sets $A$ and $B$ there exists disjoint $N \beta$-open sets $G$ and $H$ such that $A \subset G$ and $B \subset H$. This shows that $U$ is $N \beta$-normal space.

Example 5.6. Let $U=\{a, b, c, d\}, U / R_{1}=\{\{a\},\{b, d\},\{c\}\}$ $X=\{a, b\}$ and $\tau_{R}(X)=\{U, \phi,\{a\},\{a, b, d\},\{b, d\}\}$ be $a$ nano topology on $U$, we have

$N S O\left(U, \tau_{R}(X)\right)=\{U, \phi,\{a\},\{a, c\},\{b, d\},\{a, b, d\},\{b, c, d\}\}$ $N P O\left(U, \tau_{R}(X)\right)=\{U, \phi,\{a\},\{b\},\{d\},\{a, b\},\{a, d\},\{b, d\}$, $\{a, b, c\},\{a, b, d\},\{a, c, d\}$, $N \alpha O\left(U, \tau_{R}(X)\right)=\{U, \phi,\{a\},\{a, b, d\},\{b, d\}\}$ $N \beta O\left(U, \tau_{R}(X)\right)=\{U, \phi,\{a\},\{b\},\{d\},\{a, b\},\{a, c\},\{a, d\}$, $\{b, c\},\{b, d\},\{c, d\},\{a, b, c\},\{a, b, d\},\{a, c, d\},\{b, c, d\}\}$

$N S R\left(U, \tau_{R}(X)\right)=\{U, \phi,\{a\},\{a, c\},\{b, d\},\{b, c, d\}\}$

$N P R\left(U, \tau_{R}(X)\right)=\{U, \phi,\{b\},\{d\},\{a, b, c\},\{a, c, d\}\}$

$N \alpha R\left(U, \tau_{R}(X)\right)=\{U, \phi$,$\} and$

$N \beta R\left(U, \tau_{R}(X)\right)=\{U, \phi,\{a\},\{b\},\{d\},\{a, b\},\{a, c\},\{a, d\}$, $\{b, c\},\{b, d\},\{c, d\},\{a, b, c\},\{a, c, d\},\{b, c, d\}\}$.

Let $A=\{a\}$ and $B=\{c, d\}$ then it is $N \beta$-normal space but not nano-normal (resp. NS-normal, NP-normal) space.

Theorem 5.7. Every nano- $T_{1}$ space and nano-normal space is nano-regular space.

Proof. Let $U$ be nano- $T_{1}$ and nano-normal. Let $F$ be any nanoclosed set and $U \notin F \Rightarrow x \in U-F$. As $U$ is nano- $T_{1},\{\mathrm{x}\}$ is nano-closed. Thus $F$ and $\{\mathrm{x}\}$ are two disjoint nano-closed sets. Since $U$ is nano-normal, there exists disjoint nano-open sets $G$ and $H$ such that $\{x\} \subset G$ and $F \subset H$ i.e $U \in G$ and $F \subset H \Rightarrow U$ is nano-regular.

Example 5.8. From Example 3.14, let $A=\{b\}$ and $B=$ $\{a, d\}$ then it is nano-regular space but not nano-normal space (resp. nano- $T_{1}$ space)

Theorem 5.9. Every NS-T $T_{1}$ space and NS-normal space is NS-regular space.

Proof. Let $U$ be $N S-T_{1}$ and $N S$-normal. Let $F$ be any NSclosed set and $U \notin F \Rightarrow x \in U-F$. As $U$ is $N S-T_{1},\{\mathrm{x}\}$ is $N S$-closed. Thus $F$ and $\{\mathrm{x}\}$ are two disjoint $N S$-closed sets. Since $U$ is $N S$-normal, there exists disjoint $N S$-open sets $G$ and $H$ such that $\{x\} \subset G$ and $F \subset H$ i.e $U \in G$ and $F \subset H \Rightarrow U$ is $N S$-regular.

Example 5.10. From the Example 3.14, let $A=\{c\}$ and $B=$ $\{a, d\}$ then it is NS-regular space but not NS-normal space (resp. NS-T space)
Theorem 5.11. Every $N P-T_{1}$ space and NP-normal space is NP-regular space.

Proof. Let $U$ be $N P-T_{1}$ and $N P$-normal. Let $F$ be any $N P$ closed set and $U \notin F \Rightarrow U \in U-F$. As $U$ is $N P-T_{1},\{\mathrm{x}\}$ is $N P$-closed. Thus $F$ and $\{\mathrm{x}\}$ are two disjoint $N P$-closed sets. Since $U$ is $N P$-normal, there exists disjoint $N P$-open sets $G$ and $H$ such that $\{x\} \subset G$ and $F \subset H$ i.e $U \in G$ and $F \subset H \Rightarrow U$ is $N P$-regular.

Example 5.12. From the Example 3.14, let $A=\{a\}$ and $B=\{b, c\}$ then it is NP-regular space but not NP-normal space (resp. NP-T $T_{1}$ space)

Theorem 5.13. Every $N \beta-T_{1}$ space and $N \beta$-normal space is $N \beta$-regular space.

Proof. Let $U$ be $N \beta-T_{1}$ and $N \beta$-normal. Let $F$ be any $N \beta$ closed set and $U \notin F \Rightarrow U \in U-F$. As $U$ is $N \beta-T_{1},\{\mathrm{x}\}$ is $N \beta$-closed. Thus $F$ and $\{\mathrm{x}\}$ are two disjoint $N \beta$-closed sets. Since $U$ is $N \beta$-normal, there exists disjoint $N \beta$-open sets $G$ and $H$ such that $\{x\} \subset G$ and $F \subset H$ i.e $U \in G$ and $F \subset H \Rightarrow U$ is $N \beta$-regular.

Example 5.14. From the Example 3.14, let $A=\{b\}$ and $B=\{a, b, d\}$ then it is $N \beta$-regular space but not $N \beta$-normal space (resp. $N \beta-T_{1}$ space)

Theorem 5.15. : Let $U$ be $N \beta$-normal space and $Y \in N \alpha(U)$ then $Y$ is $N \beta$-normal space.

Proof. Let $U$ be $N \beta$-normal and $Y$ be a $N \alpha$-open set. Let $A_{y}$ and by disjoint $N \beta$-closed subsets of $Y$. Therefore $A_{y}=Y \cap A$ and $B_{y}=Y \cap B$ where $A$ and $B$ are $N \beta$-closed subsets of $U$ such that $A \cap B=\phi$. As $U$ is $N \beta$-normal, there exists disjoint $N \beta$-open subsets $G$ and $H$ of $U$ such that $A \subset G$ and $B \subset H \Rightarrow Y \cap A \subset Y \cap G$ and $Y \cap B \subset Y \cap H$ where $Y \cap G$ and $Y \cap H$ are $N \beta$-open subsets of $Y$ such that $(Y \cap G) \cap(Y \cap H)=$ $Y \cap(G \cap H)=\phi$. Hence $Y$ is $N \beta$-normal.

Theorem 5.16. A nano topological space $\left(U, \tau_{R}(X)\right)$ is $N \beta$ normal iff for any $N \beta$-closed set $F$ and $N \beta$-open set $G$ containing $F$ there exists a $N \beta$-open set $K$ such that $F \subset K$ and $N \operatorname{Nl}(K) \subset G$.

Proof. Let $U$ be $N \beta$-normal and $F$ be $N \beta$-closed set and $G$ be $N \beta$-open such that $F \subset G$. This shows that $(U-G) \cap F=\phi$, as $U$ is $N \beta$-normal corresponding to these two disjoint $N \beta$ closed sets $U-G$ and $F$ there exists disjoint $N \beta$-open sets $H$ and $K$ such that $U-G \subset H$ and $F \subset K \ldots \ldots \ldots . .(1)$ Now $U-G \subset H \Rightarrow U-H \subset G$.........(2) Also $H \cap K=\phi \Rightarrow K \subset$ $U-H . N \beta c l(K) \subset N \beta C l(U-H)=U-H \subset G$ From (1), $N \beta c l(K) \subset G \ldots \ldots . . .(3)$ From (1) and (3) we conclude that $K$ is $N \beta$-open set such that $F \subset K$ and $N \beta \operatorname{cl}(K) \subset G$.

Conversely: For any $N \beta$-closed set $F$ and $N \beta$-open set $G$ containing $F$ there exists $N \beta$-open set $K$ such that $F \subset K$ and $N \beta \operatorname{cl}(K) \subset G$. We have to prove that $U$ is $N \beta$-normal. Let $A$ and $B$ be any two disjoint $N \beta$-closed sets of $U$. Therefore $A \subset U-B$. Thus $A$ is $N \beta$-closed set and $U-B$ is $N \beta$-open set 
containing $A$, then by hypothesis there exists $N \beta$-open set $K$ such that $A \subset K$ and $N \beta c l(K) \subset U-B \Rightarrow B \subset U-N \beta c l(K)$. As $K$ is $N \beta$-open, $N \beta c l(K)$ is $N \beta$-regular $\Rightarrow U-N \beta c l(K)$ is $N \beta$-open. Also $U-N \beta \operatorname{cl}(K) \subset U-K \Rightarrow(U-N \beta c l(K)) \cap$ $K \subset(U-K) \cap K=\phi$ i.e $(U-N \beta c l(K)) \cap K=\phi$. Thus for any two disjoint $N \beta$-closed sets $A$ and $B$ there exists disjoint $N \beta$-open sets $K$ and $U-N \beta c l(K)$ such that $A \subset K$ and $B \subset$ $U-N \beta \operatorname{cl}(K)$. Hence $U$ is $N \beta$-normal.

Theorem 5.17. For a nano topological space $U$ the following are equivalent:

1. $U$ in $N \beta$-normal.

2. For every pair of nano open sets $G$ and $H$ whose union is $U$, there exists $N \beta$-closed sets $A$ and $B$ such that $A \subset G, B \subset H$ and $A \cup B=U$.

3. For every nano closed set $L$ and every nano open set $K$ containing $H$, there exists a $N \beta$-open set $G$ such that $L \subseteq G \subseteq N \beta c l(G) \subseteq K$.

Proof. (1) $\Rightarrow(2)$ Let $G$ and $H$ be pair of nano open sets in a $N \beta$ - normal space $U$ such that $U=G \cup H$. Then $U / G$ and $U / H$ are disjoint nano closed sets. Since $U$ is $N \beta$-normal there exists disjoint $N \beta$ - normal sets $G_{1}$ and $H_{1}$ such that $U / G \subset G_{1}$ and $U / H \subset H_{1}$. Let $A=U / G_{1}$ and $B=U / H_{1}$. Then $A$ and $B$ are $N \beta$ - closed sets such that $A \subseteq G, B \subseteq H$ and $A \cup B=U$.

(2) $\Rightarrow$ (3) Let $H$ be a nano closed set and $K$ be an nano open set containing $L$. Then $U / L$ and $K$ are nano open sets whose union is $U$. Then by (2), there exists $N \beta$-closed sets $M_{1}$ and $M_{2}$ such that $M_{1} \subseteq U / L$ and $M_{2} \subset K$ and $M_{1} \cup M_{2}=U$. Then $L \subseteq U / M_{1}, U / K \subseteq U / M_{2}$ and $U / M_{1} \cap U / M_{2}=\phi$.

Let $G=U / M_{1}$ and $H=U / M_{2}$. Then $G$ and $H$ are disjoint $N \beta$ - open sets such that $L \subseteq G \subseteq U / H \subseteq K$. As $U / H$ is $N \beta$-closed set, we have $N \beta \operatorname{cl}(G) \subseteq U / H$ and $L \subseteq G \subseteq$ $N \beta c l(G) \subseteq K$.

(3) $\Rightarrow$ (1) Let $L_{1}$ and $L_{2}$ be any two disjoint closed sets of $U$. Put $K=U / L_{2}$, then $L_{2} \cap K=\phi, L_{1} \subseteq K$ where $K$ is nano-open set. Then by (3), there exists a $N \beta$-open sets $G$ of $U$ such that $L_{1} \subseteq G \subseteq N \beta c l(G) \subseteq K$. It follows that $L_{2} \subseteq U / N \beta c l(G)=$ $H$, say, then $H$ is $N \beta$-open and $G \cap H=\phi$. Hence $L_{1}$ and $L_{2}$ are seperated by $N \beta$-open sets $G$ and $H$. Therefore $U$ is $N \beta$-normal.

\section{References}

[1] Lellis Thivagar and Richard.C, On nano forms of weekly open sets, International Journal of Mathematics and Statistics Invention. 1 (1)(2013), 31 - 37.

[2] Pawalk.Z, Rough sets, Theoretical Aspects of Reasoning about Data, Kluwer Academic Publishers, Boston, 1991

[3] Sathishmohan.P, Rajendran.V, Dhanasekaran.P.K and Vignesh Kumar.C, More on nano pre-neighbourhoods in nano topological-spaces(Communicated)
[4] Sathishmohan.P, Rajendran.V, Dhanasekaran.P.K and Brindha.S, Further properties of nano pre- $T_{0}$, nano pre- $T_{1}$ and nano pre- $T_{2}$ spaces(Communicated)

[5] Sathishmohan.P, Rajendran.V, Dhanasekaran.P.K, More on nano pre-regular spaces in nano topological space, Journal of Applied Science and Computations, Volume 5, 10, 2018.

[6] Sathishmohan.P, Rajendran.V, Vignesh Kumar.C and Dhanasekaran.P.K, On $N \beta$ neighbourhoods on nano topological spaces, Malaya Journal of Mathematik, Vol.6, No.1, 2018, 294 -298,

[7] Sathishmohan.P, Rajendran.V and Vignesh Kumar.C, A few separation axioms in nano topological space (communicated).

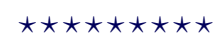

$$
\begin{aligned}
& \text { ISSN(P):2319 - } 3786 \\
& \text { Malaya Journal of Matematik } \\
& \text { ISSN(O):2321 - } 5666
\end{aligned}
$$

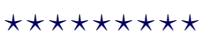

